\title{
The identification of human aldo-keto reductase AKR7A2 as a novel cytoglobin- binding partner
}

\author{
Xin Li, Shanshan Zou, Zhen Li, Gaotai Cai, Bohong Chen, Ping Wang and Wenqi Dong*
}

\author{
* Correspondence: \\ dongwq63@263.net \\ Department of Biopharmaceutics, \\ School of Laboratory Medicine and \\ Biotechnology, Southern Medical \\ University, 1838 North Guangzhou \\ Avenue, Guangzhou 510515, China
}

\begin{abstract}
Cytoglobin (CYGB), a member of the globin family, is thought to protect cells from reactive oxygen and nitrogen species and deal with hypoxic conditions and oxidative stress. However, its molecular mechanisms of action are not clearly understood. Through immunoprecipitation combined with a two-dimensional electrophoresis-mass spectrometry assay, we identified a CYGB interactor: aldo-keto reductase family 7 member A2 (AKR7A2). The interaction was further confirmed using yeast two-hybrid and co-immunoprecipitation assays. Our results show that AKR7A2 physically interacts with CYGB.
\end{abstract}

Keywords: CYGB, AKR7A2, Protein-protein interactions, Yeast two-hybrid assay, Co-immunoprecipitation, 2-DE, Oxidative stress

\section{Introduction}

Cytoglobin (CYGB), which is a member of the globin family, was discovered more than a decade ago in a proteomic screen of fibrotic liver [1]. It was originally named STAP (stellate activating protein). Human CYGB is a 190-amino acid, 21-kDa protein [2], encoded by a single copy gene mapped at the $17 \mathrm{q} 25.3$ chromosomal segment [3]. It has a compact helical conformation, giving it the ability to bind to heme, which allows reversible binding of gaseous, diatomic molecules, including oxygen $\left(\mathrm{O}_{2}\right)$, nitric oxide (NO) and carbon monoxide ( $\mathrm{CO})$, just like hemoglobin $(\mathrm{Hb})$, myoglobin $(\mathrm{Mb})$ and neuroglobin (Ngb) [4]. Unlike those family members, which are respectively localized in the erythrocytes, muscles and neurons and neuroendocrine tissue, CYGB is expressed across a broad spectrum of vertebrate organs and tissues with varying expression levels. It is found predominantly in the fibroblasts of connective tissue and in fibroblast-related cell lineages, such as chondroblasts, osteoblasts, hepatic stellate cells (HSCs) and myofibroblasts, and it may play a role in fibrotic organ disorder $[3,5]$.

Given its similarity to other globins (particularly $\mathrm{Mb}$ and $\mathrm{Ngb}$ ), several respiratory and stress-related activities for CYGB have been considered. These include oxygen storage, transport and sensing, terminal oxidase activity, nitric oxide dioxygenase activity [6], and scavenging of reactive oxygen species (ROS) [7]. It is also thought to protect cells from reactive oxygen and nitrogen species and deal with hypoxic conditions and oxidative stress in the cells [8]. CYGB is associated with various diseases,

(c) The Author(s). 2016 Open Access This article is distributed under the terms of the Creative Commons Attribution 4.0 International License (http://creativecommons.org/licenses/by/4.0/), which permits unrestricted use, distribution, and reproduction in any medium, provided you give appropriate credit to the original author(s) and the source, provide a link to the Creative Commons license, and indicate if changes were made. The Creative Commons Public Domain Dedication waiver (http://creativecommons.org/ publicdomain/zero/1.0/) applies to the data made available in this article, unless otherwise stated. 
such as organ fibrosis in the liver, kidney and pancreas, glaucoma, gastroesophageal reflux disease, and several neurodegenerative disorders. Moreover, considerable evidence suggests that CYGB may function as a tumor suppressor [9].

There has been no definite evidence to support the participation of CYGB in any protein-protein interactions. Although previous studies using the yeast two-hybrid assay and affinity capture discovered that ATPase type 13A2 (ATP13A2) DNA damageinducible 1 homolog 1 (DDI1) and SH3-domain kinase binding protein 1 (SH3KBP1) were candidate genes for interaction with $\mathrm{CYGB}$, there was no further evidence to support this hypothesis.

In this study, we focused our investigation on possible novel binding partners interacting with CYGB. We used immunoprecipitation combined with a two-dimensional electrophoresis-mass spectrometry assay. Since CYGB has a high expression level in hepatic stellate cells (HSCs) and may play a role in fibrotic organ disorder, we choose LX-2 cells to screen the CYGB-interacting proteins. The putative interactor was further confirmed using the yeast strain Y2HGold in a yeast two-hybrid assay and using mammalian cells in a co-immunoprecipitation assay.

\section{Materials and methods}

Plasmid constructions

The full-length cDNA of CYGB was inserted into pcDNA3.0-FLAG and pGBKT7 to respectively generate pcDNA3.0-FLAG-CYGB and pGBKT7-CYGB. The full-length cDNA of AKR7A2 was inserted into pCMV-MYC and pGADT7 to respectively generate pCMV-MYC-AKR7A2 and pGADT7-AKR7A2. All of the cDNA was obtained from human hepatic stellate cells (LX-2) via RT-PCR. The primers are listed in Table 1. All constructs were confirmed via sequence analysis.

\section{Cell culture and transfection}

LX-2 cells (donated by Dr. Scott Friedman, School of Mount Sinai, USA) and HEK293T cells (preserved in our laboratory) were both maintained in Dulbecco's modified Eagle's medium (Hyclone) containing $10 \%$ fetal bovine serum (Hyclone), 100 units $/ \mathrm{ml}$ penicillin and $100 \mu \mathrm{g} / \mathrm{ml}$ streptomycin.

One day before transfection, the LX-2 cells were seeded into 6-well culture plates. When the cells reached $\sim 80 \%$ confluence, the pcDNA3.0-FLAG or pcDNA3.0-FLAG-CYGB

Table 1 Primers used in this study

\begin{tabular}{ll}
\hline Constructs & Primer sequence (5'-3') \\
\hline pcDNA3.0-FLAG-CYGB & F: AGCTAGATTACAAGGATGACGACGA \\
& TAAGATGGAGAAAG \\
pGBKT7-CYGB & R: CGGAATTCCTACGGCCCCGAAGAGG \\
& F: CGGATTCATGGAGACAGGCGAG \\
pCMV-MYC-AKR7A2 & R: AAAACTGCAGCTACGGCCCCGAAGAGG \\
& F: CGAATTCAAATGCTGAGTGCCGCGTCT \\
pGADT7-AKR7A2 & R: CCGACTCGAGCTAGCGGAAGTAGTTGGGA \\
& F: CGAATTCATGCTGAGTGCCGCGTCT \\
& R: CCGACTCGAGCTAGCGGAAGTAGTTGGGA \\
\hline
\end{tabular}


vector was transfected into the cells using Lipofectamine 2000 (Invitrogen) according to the manufacturer's protocol. HEK293T cells were co-transfected using pCMV-MYC-AKR7A2 together with pcDNA3.0-FLAG or pcDNA3.0-FLAG -CYGB vector. All cells were cultured at $37{ }^{\circ} \mathrm{C}$ with $5 \% \mathrm{CO}_{2}$.

\section{Immunoprecipitation}

Whole cell extracts of transfected LX-2 cells were prepared $48 \mathrm{~h}$ post-transient transfection in a mild lysis buffer consisting of $20 \mathrm{mM}$ Tris, $150 \mathrm{mM} \mathrm{NaCl}, 2 \%$ glycerol, 1.6 mM EDTA, $0.5 \%$ Triton X-100 and a protease inhibitor cocktail. The supernatant was collected, incubated with ANTI-FLAG M2 Affinity Gel (Sigma) overnight at $4{ }^{\circ} \mathrm{C}$ and then centrifuged for $30 \mathrm{~s}$ at $7500 \mathrm{~g}$. The precipitates were washed with $1 \mathrm{ml} \mathrm{IP}$ washing buffer A (20 mM Tris, $150 \mathrm{mM} \mathrm{NaCl}$ ) three times and $1 \mathrm{ml}$ IP washing buffer B (20 mM Tris) twice. Finally, the precipitates were eluted with $300 \mu \mathrm{l}$ IP elution buffer (6 M urea, $2 \mathrm{M}$ thiourea, $4 \%$ CHAPS, $0.3 \%$ ampholytes, $0.002 \%$ bromophenol blue), and the eluted proteins were prepared for two-dimensional electrophoresis.

\section{Two-dimensional gel electrophoresis and mass spectrometry}

Two-dimensional gel electrophoresis (2-DE) was performed according to the Bio-Rad manual, with the first dimension isoelectrofocusing carried out on 17-cm IPG strips (pH3-10 NL, Bio-Rad) and the second dimension separated by $10 \%$ SDS-PAGE. Before the first dimension isoelectrofocusing, the protein samples were applied to IPG strips for $2 \mathrm{~h}$ passive rehydration and $12 \mathrm{~h}$ active rehydration loading at $50 \mathrm{~V}$ and $20{ }^{\circ} \mathrm{C}$. The first dimension isoelectrofocusing was carried out using Protean IEF Cell (Bio-Rad) with six steps: $250 \mathrm{~V}$ for $1 \mathrm{~h}, 500 \mathrm{~V}$ for $1 \mathrm{~h}, 1000 \mathrm{~V}$ for $1 \mathrm{~h}$ and $10000 \mathrm{~V}$ for $3 \mathrm{~h}$, then $10000 \mathrm{~V}$ at 68000 Vhour, and finally $500 \mathrm{~V}$ for $3 \mathrm{~h}$. All steps were at $20^{\circ} \mathrm{C}$. After isoelectrofocusing, the proteins were in-gel equilibrated in two steps: with a $50 \mathrm{mM}$ Tris buffer containing $6 \mathrm{M}$ urea, $2 \%$ SDS $(w / v), 10 \%$ glycerol $(v / v)$ and $2.5 \%$ DTT $(w / v)$ for $15 \mathrm{~min}$; and with a $50 \mathrm{mM}$ Tris buffer containing $6 \mathrm{M}$ urea, $2 \%$ SDS $(w / v), 10 \%$ glycerol $(v / v)$ and $3.3 \%$ iodoacetamide $(w / v)$ for $15 \mathrm{~min}$. The equilibrated IPG strips were then transferred onto two $10 \%$ polyacrylamide slab gels and the SDS-PAGE was carried out in two steps: $10 \mathrm{~mA} / \mathrm{gel} / 17 \mathrm{~cm}$ for $1 \mathrm{~h}$ and $28 \mathrm{~mA} / \mathrm{gel} / 17 \mathrm{~cm}$ for $9 \mathrm{~h}$. Finally, the separated proteins were visualized via silver staining and image analysis was performed using PDQuest 2-D analysis software V8.0.1 (Bio-Rad). In-gel digestion and MALDI-TOF/MS/MS analysis was performed at the Beijing Genomics Institute in Shenzhen, China.

\section{Yeast two-hybrid assay}

The Matchmaker Gold Yeast Two-Hybrid System (Clontech) was used to identify the interaction between CYGB and AKR7A2. Human CYGB CDNA was inserted into the pGBKT7 vector (Clontech) to generate pGBKT7-CYGB as the bait. Human AKR7A2 cDNA was inserted into the pGADT7 vector (Clontech) to generate pGADT7-AKR7A2 as the prey. Using the small-scale transformation procedure given in the Matchmaker Gold Yeast Two-Hybrid System User Manual, we co-transformed $100 \mathrm{ng}$ of each of the following pairs of vectors into Y2HGold Competent Cells: Empty pGBKT7 + Empty pGADT7, pGBKT7-CYGB + Empty pGADT7, Empty pGBKT7 + pGADT7-AKR7A2, pGBKT7-CYGB + pGADT7-AKR7A2, pGBKT7-53 + pGADT7-T as a positive control, 
and pGBKT7-lam + pGADT7-T as a negative control. The transformed Y2HGold Cells were then plated on SD/-Leu/-Trp Agar and SD/-Ade/-His/-Leu/-Trp/X-a-Gal/AbA agar.

\section{Co-immunoprecipitation}

HEK 293 T cells were co-transfected using pCMV-MYC-AKR7A2 together with pcDNA3.0-FLAG or pcDNA3.0-FLAG-CYGB vectors. Following transient transfection for $48 \mathrm{~h}$, the cells were lysed in a mild lysis buffer consisting of $20 \mathrm{mM}$ Tris, $150 \mathrm{mM}$ $\mathrm{NaCl}, 2$ \% glycerol, $1.6 \mathrm{mM}$ EDTA, $0.5 \%$ Triton X-100 and a protease inhibitor cocktail. The supernatant was collected and incubated with ANTI-FLAG M2 Affinity Gel (Sigma) overnight at $4{ }^{\circ} \mathrm{C}$, and then centrifuged for $30 \mathrm{~s}$ at $7600 \mathrm{~g}$. The precipitates were washed with $600 \mu \mathrm{l}$ IP washing buffer $(20 \mathrm{mM}$ Tris, $150 \mathrm{mM} \mathrm{NaCl})$ four times. After that, the precipitates were eluted with $2 \times$ sample buffer consisting of $125 \mathrm{mM}$ Tris $\mathrm{HCl}(\mathrm{pH}$ 6.8) with $4 \%$ SDS, $20 \%(v / v)$ glycerol and $0.004 \%$ bromphenol blue, and boiled for $3 \mathrm{~min}$. Finally, the samples were centrifuged for $30 \mathrm{~s}$ at $7600 \mathrm{~g}$ and the supernatant was prepared for loading on SDS-PAGE and immunoblotting.

\section{Results}

\section{Identification of AKR7A2 as a possible CYGB interactor}

In order to identify the interactors of CYGB, we carried out an immunoprecipitation experiment followed by two-dimensional gel electrophoresis (2-DE) and mass spectrometry analysis. FLAG-tagged CYGB protein and FLAG-tag alone were expressed in LX-2 cells and precipitated using ANTI-FLAG M2 Affinity Gel. The proteins co-precipitated with FLAG-tagged CYGB protein or FLAG-tag were used for the 2-DE analysis, the images from which are shown in (Fig. 1). The unique spot present in the experimental gel but not in the control was subjected to in-gel digestion and MALDI-TOF/MS/MS analysis. We then performed a search based on peptide mass fingerprint matching in the Swiss-Prot protein database using the Mascot search engine. Aldo-keto reductase family 7 member a2 (AKR7A2) was identified as a candidate with a significant protein score $(p<0.05)$, and the results of the mass spectrometry are shown in (Table 2) and (Fig. 2).

\section{AKR7A2 interacted with CYGB in the yeast strain Y2HGold}

To confirm the interaction between AKR7A2 and CYGB, we carried out a yeast twohybrid assay with Y2HGold competent cells. As shown in (Fig. 3), Y2HGold cells grew on the $\mathrm{SD} /$-Leu/-Trp agar plate, indicating that all the vector pairs were successfully co-transformed into Y2HGold competent cells. On the SD/-Ade/-His/-Leu/-Trp/X-aGal/AbA agar plate, only the Y2HGold cells co-transformed with pGBKT7-CYGB, pGADT7-AKR7A2 or the positive control could grow and turn blue, indicating that AKR7A2 interacted with CYGB in Y2HGold.

\section{AKR7A2 interacted with CYGB in HEK293T cells}

For further verification of the interaction between AKR7A2 and CYGB in mammalian cells, we performed a co-immunoprecipitation experiment. As shown in (Fig. 4a) and (Fig. 4b), FLAG-tagged CYGB protein and MYC-tagged AKR7A2 protein could be stably and highly expressed in HEK $293 \mathrm{~T}$ cells, which was significant to the following co- 


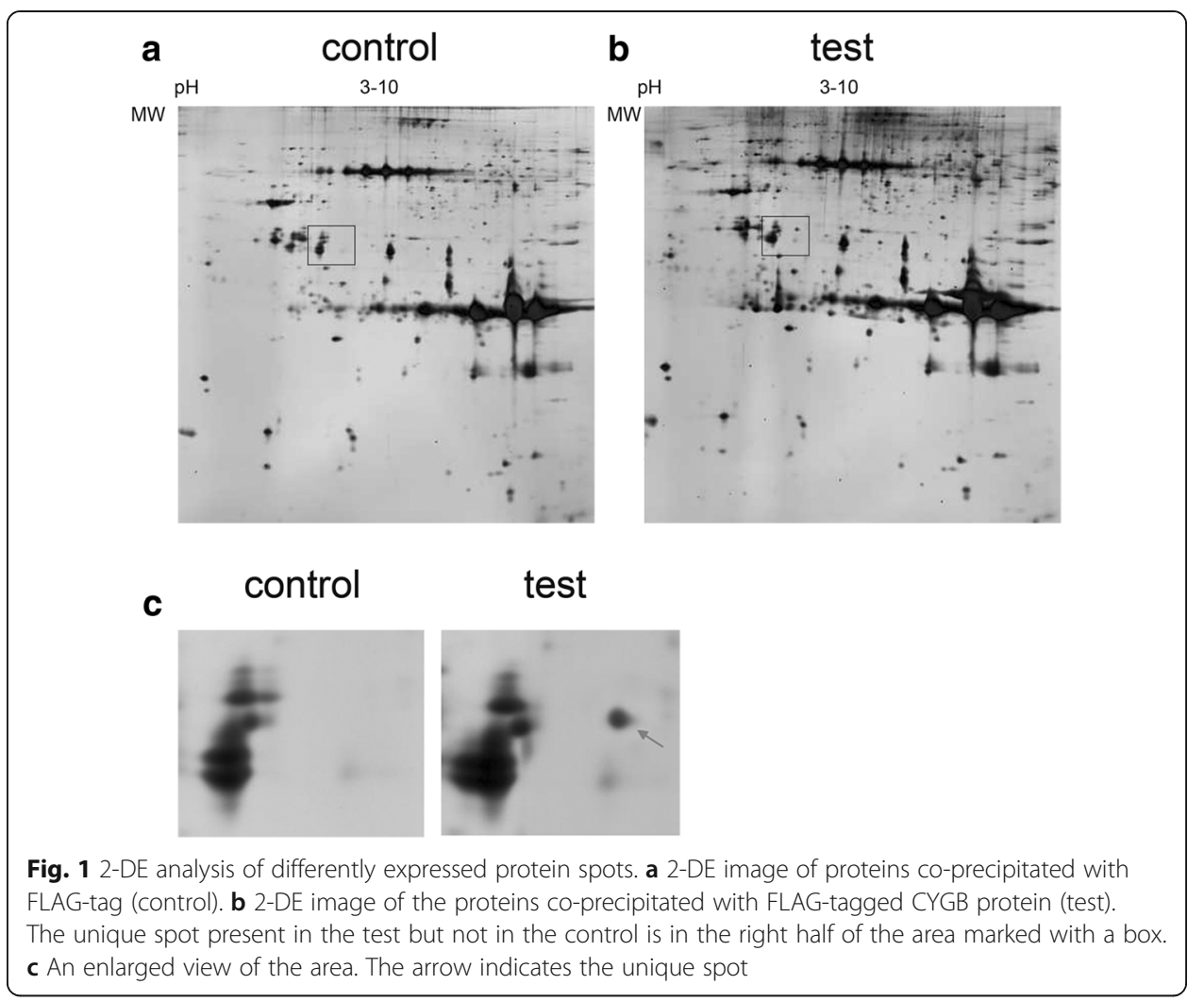

immunoprecipitation experiment. MYC-tagged AKR7A2 protein was detected in the precipitates from cell lysates co-transfected with both proteins (Fig. 4c, lane 4) but not from cell lysates co-transfected with MYC-tagged AKR7A2 protein and FLAG empty vector (Fig. 4c, lane 3). In summary, MYC-tagged AKR7A2 protein was efficiently precipitated by FLAG-CYGB fusion protein but not by FLAG alone, indicating that AKR7A2 interacts with CYGB in mammalian cells.

\section{Discussion}

AKR7A2 is a member of the aldo/keto reductase (AKR) superfamily and AKR7 family. AKRs are a superfamily of $\mathrm{NADP}(\mathrm{H})$-dependent enzymes that reduce aldehydes and ketones to alcohols [10]. Several AKR have been suggested to be under the transcriptional control of nuclear factor erythroid 2-related factor 2 (Nrf2) [11]. The Keap1Nrf2-ARE pathway is of critical importance in cellular defense against stress, and Nrf2 is reported to mediate the gene expression of AKR7A2 in response to methyl glyoxal

Table 2 Mass spectrometry results of the unique spot in the 2-DE image

\begin{tabular}{|c|c|c|c|c|c|c|}
\hline Rank & Protein name & Accession no. & $\begin{array}{l}\text { Protein } \\
\text { score }\end{array}$ & $\begin{array}{l}\text { Protein } \\
\text { score C.I. \% }\end{array}$ & $\begin{array}{l}\text { Protein } \\
\text { MW }\end{array}$ & Protein PI \\
\hline 1 & $\begin{array}{l}\text { Aflatoxin B1 aldehyde } \\
\text { reductase member } 2 \text { (AKR7A2) }\end{array}$ & sp|O43488|AKR7A2_HUMAN & 59 & 95.568 & 40019.9 & 6.7 \\
\hline 2 & $\begin{array}{l}\text { Small ubiquitin-related } \\
\text { modifier } 1 \text { (SUMO1) }\end{array}$ & sp|P63165|SUMO1_HUMAN & 24 & 48.644 & 11606.7 & 5.35 \\
\hline 3 & $\begin{array}{l}\text { Dedicator of cytokinesis } \\
\text { protein } 11 \text { (DOCK11) }\end{array}$ & sp|Q5JSL3|DOCK11_HUMAN & 19 & 2.369 & 240140.6 & 7.87 \\
\hline
\end{tabular}




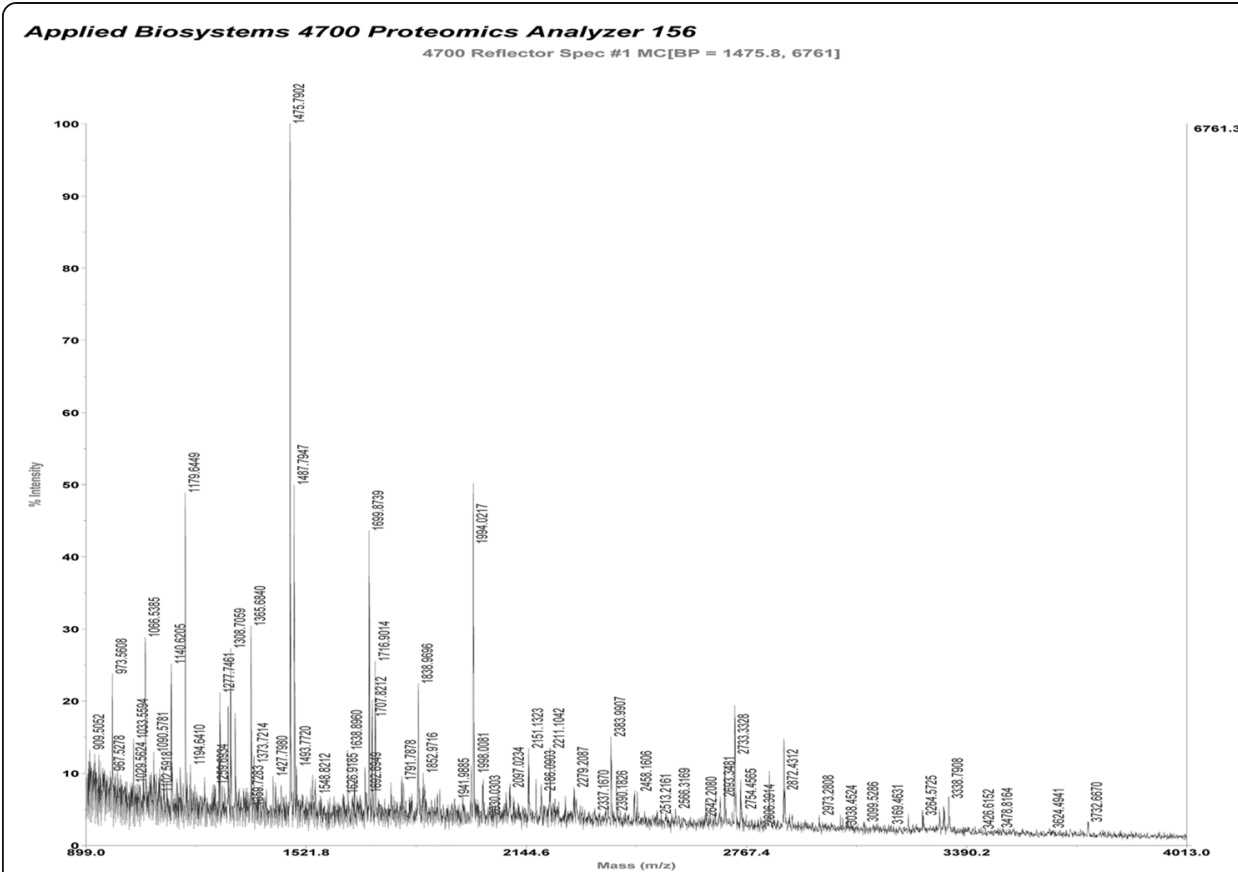

Fig. 2 MALDI-TOF/MS/MS results from the analysis of the unique spot in the 2-DE image

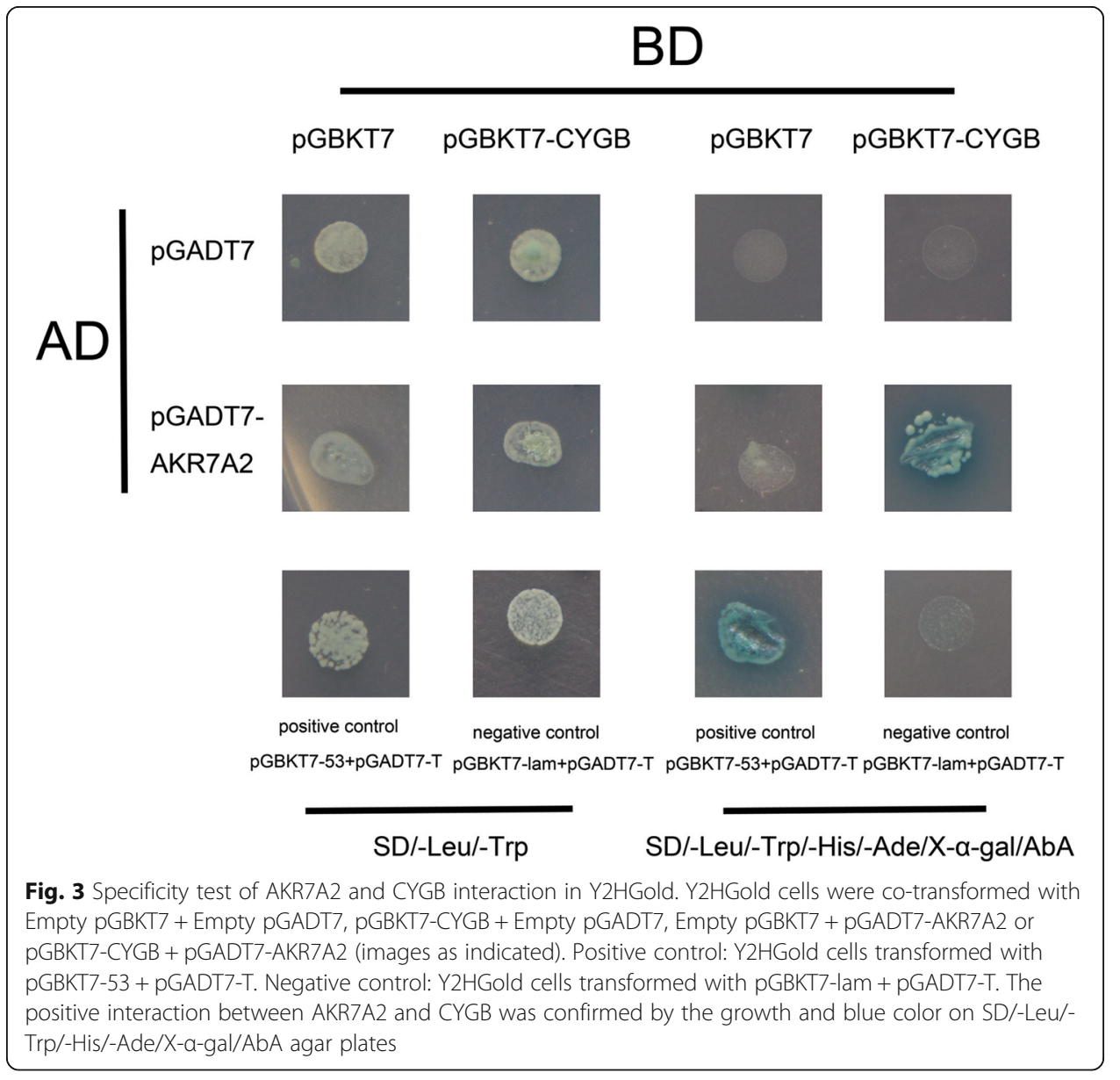




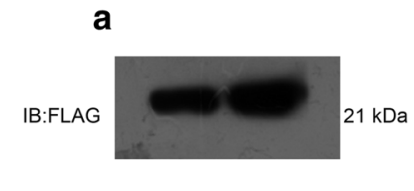

b

IB:MYC

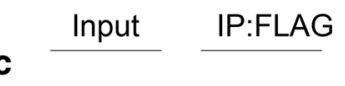

IB:FLAG

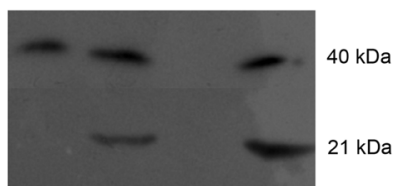

PCDNA3.0-FLAG $\begin{array}{lllll}\text { PCDNA3.O-FLAG-CYGB } & - & + & - & + \\ \text { PCMV-MYC-AKR7A2 } & + & + & + & +\end{array}$

Fig. 4 Confirmation of the interaction between AKR7A2 and CYGB in HEK 293 T cells. a The expression of FLAG-tagged CYGB protein in HEK 293 T cells. b The expression of MYC-tagged AKR7A2 protein in HEK 293 T cells. c Co-immunoprecipitation of AKR7A2 and CYGB. HEK293T cells were co-transfected using pCMVMYC-AKR7A2 together with pcDNA3.0-FLAG or pCDNA3.0-FLAG-CYGB vectors and then subjected to immunoprecipitation with ANTI-FLAG M2 Affinity Gel, followed by immunoblotting with anti-MYC and anti-FLAG antibody

(MG) [12]. AKR7A2 is known to be present in a range of tissues, including the liver, kidneys and brain [13]. Previous studies have shown that the AKR7A2 enzyme is catalytically active toward aldehydes arising from lipid peroxidation, suggesting a potential protective role against the consequences of oxidative stress, and representing an important detoxification route in mammals. It may have a role in the defense against ROS, an important factor in disease states that have an oxidative stress component [14], which is similar to CYGB.

Since both AKR7A2 and CYGB are involved in ROS scavenging activity and in the response to oxidative stress, we will explore the possible physiological meaning of this interaction in our future studies.

\section{Conclusion}

In this study, we identified a putative CYGB-interacting protein, AKR7A2, and evidenced its physical interaction with CYGB. These results may provide some clues to the hitherto unknown molecular mechanism of CYGB.

\section{Abbreviations}

2-DE: Two-dimensional gel electrophoresis; AKR: Aldo-keto reductases; AKR7A2: Aldo-keto reductase family 7 Member A2; ARE: Antioxidant response element; ATP13A2: ATPase type 13A2; Cygb: Cytoglobin; DDI1: DNA damage-inducible 1 homolog 1; MG: Methyl glyoxal; Nrf2: Nuclear factor erythroid 2-related factor 2; RNS: Reactive nitrogen species; ROS: Reactive oxygen species; SD: Selection medium; SH3KBP1: SH3-domain kinase binding protein 1; Y2H: Yeast two-hybrid

\section{Acknowledgements}

We would like to acknowledge anonymous reviewers and our academic editors for their constructive suggestions on the manuscript.

\section{Funding}

This research was supported by the Guangdong Province Science and Technology Plan Project (No. 2010B031500012, No. 2013A022100027) and the Guangzhou Science and Technology Project (No. 201510010104).

Availability of data and materials

Data and materials listed in the paper are available in our laboratories.

Authors' contributions

$\mathrm{XL}$ planned and performed the experiments, and wrote the manuscript. SZ, ZL, GC, BC, and PW performed the experiments. WD designed the study and revised the manuscript. All authors read and approved the final manuscript. 
Consent for publication

Not applicable.

Ethics approval and consent to participate

Not applicable.

Received: 20 November 2015 Accepted: 14 March 2016

Published online: 24 October 2016

\section{References}

1. Asahina K, Kawada N, Kristensen DB, Nakatani K, Seki S, Shiokawa M, Tateno C, Obara M, Yoshizato K.

Characterization of human stellate cell activation-associated protein and its expression in human liver. BBA-Gen Subjects. 2002;1577:471-5.

2. Kawada N, Kristensen DB, Asahina K, Nakatani K, Minamiyama Y, Seki S, Yoshizato K. Characterization of a stellate cell activation-associated protein (STAP) with peroxidase activity found in rat hepatic stellate cells. J Biol Chem. 2001;276:25318-23.

3. Oleksiewicz U, Liloglou T, Field JK, Xinarianos G. Cytoglobin: biochemical, functional and clinical perspective of the newest member of the globin family. Cell Mol Life Sci. 2011;68:3869-83.

4. Wajcman H, Kiger L, Marden MC. Structure and function evolution in the superfamily of globins. C R Biol. 2009:332:273-82.

5. Nakatani K, Okuyama H, Shimahara Y, Saeki S, Kim DH, Nakajima YJ, Seki S, Kawada N, Yoshizato K. Cytoglobin/STAP, its unique localization in splanchnic fibroblast-like cells and function in organ fibrogenesis. Lab Invest. 2004;84:91-101.

6. Li H, Hemann C, Abdelghany TM, El-Mahdy MA, Zweier JL. Characterization of the mechanism and magnitude of cytoglobin-mediated nitrite reduction and nitric oxide generation under anaerobic conditions. J Biol Chem. 2012; 287:36623-33

7. Fordel E, Thijs L, Moens L, Dewilde S. Neuroglobin and cytoglobin expression in mice. Evidence for a correlation with reactive oxygen species scavenging. FEBS J. 2007;274:1312-7.

8. Trent JT, Trent 3rd JT, Hargrove MS. A ubiquitously expressed human hexacoordinate hemoglobin. J Biol Chem. 2002;277:19538-45

9. Fang J, Ma I, Allalunis-Turner J. Knockdown of cytoglobin expression sensitizes human glioma cells to radiation and oxidative stress. Radiat Res. 2011;176:198-207.

10. Barski OA, Tipparaju SM, Bhatnagar A. The aldo-keto reductase superfamily and its role in drug metabolism and detoxification. Drug Metab Rev. 2008;40:553-624.

11. Wilmes A, Lenard MO, Jennings P. Nrf2 inducibility of aldo-keto reductases. Toxicol Lett. 2013;221:39.

12. Li D, Ma S, Ellis EM. Nrf2-mediated adaptive response to methyl glyoxal in HepG2 cells involves the induction of AKR7A2. Chem Biol Interact. 2015;234:366-71.

13. Tania O, Linda SI, David JH, John DH. Major differences exist in the function and tissue-specific expression of human aflatoxin B1 aldehyde reductase and the principal human aldo-keto reductase AKR1 family members. Biochem J. 1999:343:487-504.

14. Li D, Ferrari M, Ellis EM. Human aldo-keto reductase AKR7A2 protects against the cytotoxicity and mutagenicity of reactive aldehydes and lowers intracellular reactive oxygen species in hamster V79-4 cells. Chem Biol Interact. 2012;195:25-34.

\section{Submit your next manuscript to BioMed Central and we will help you at every step:}

- We accept pre-submission inquiries

- Our selector tool helps you to find the most relevant journal

- We provide round the clock customer support

- Convenient online submission

- Thorough peer review

- Inclusion in PubMed and all major indexing services

- Maximum visibility for your research

Submit your manuscript at www.biomedcentral.com/submit 